Relations industrielles

Industrial Relations

\title{
Group Thinking and Conference Leadership, William E. Utterback, New York : Holt, Rinehart and Winston, Inc., 1964, $244 \mathrm{pp}$.
}

\section{Jean Michaud}

Volume 20, numéro 1, 1965

URI : https://id.erudit.org/iderudit/027553ar

DOI : https://doi.org/10.7202/027553ar

Aller au sommaire du numéro

Éditeur(s)

Département des relations industrielles de l'Université Laval

ISSN

0034-379X (imprimé)

1703-8138 (numérique)

Découvrir la revue

Citer ce compte rendu

Michaud, J. (1965). Compte rendu de [Group Thinking and Conference

Leadership, William E. Utterback, New York : Holt, Rinehart and Winston, Inc., 1964, 244 pp.] Relations industrielles / Industrial Relations, 20(1), 194-195.

https://doi.org/10.7202/027553ar

Tous droits réservés @ Département des relations industrielles de l'Université Laval, 1965
Ce document est protégé par la loi sur le droit d'auteur. L'utilisation des services d'Érudit (y compris la reproduction) est assujettie à sa politique d'utilisation que vous pouvez consulter en ligne.

https://apropos.erudit.org/fr/usagers/politique-dutilisation/ 


\section{RECENSIONS - BOOK REVIEWS}

Gestion prospective de l'entreprise "por Wilfrid Brown, Dunod Paris, 1964. 379 pages. Traduit de l'anglais par Philippe de Coulon et Jacques Biadi

Vous ne trouverez pas dans ce volume une conception a avant-gardiste, de l'administration de l'entreprise, mais bien une démarche intellectuelle qui a permis ò l'outeur, président de lo Glacier Co., de visualiser en des termes simples et précis la marche octuelle de son entreprise. C'est réellement un tour de force pour un praticien de d'ossigner lo tôche d'aller ou delà de la simple observotion jusqu'à la définition de concepts pour orriver à se représenter mentolement ce que veut occomplir son entreprise et comment elle le veut. Brown invite les dirigeants d'entreprise, non pas à appliquer ses solutions, mais bien à un travail de réflexion qu'il o lui-même accompli. II fout ajouter cependant qu'il a bénéficé de l'aide de Tavistock Institute et, en particulier, d'Eliot Jocques. Ce dernier est de plus en plus connu par sa tentative d'élaborer un système permettant d'évoluer objectivement lo responsabilité des dirigeants.

L'onalyse de Brown part d'une observation des structures de l'organisation. Il en distingue quatre: l'organisation explicite, de fait, supposée et requise.

En étudiont comment les spécialistes s'intégraient de fait dans les structures, il a constaté l'inefficacité dans la conduite des opérations sur la base de l'autorité fonctionnelle. Ce fait l'a poussé ò implanter l'orgonisation requise pour permettre oux spécialistes d'exercer efficacement une autorité fonctionnelle à l'intérieur de frontières très précises.

Brown distingue oussi trois systèmes dans l'entreprise. a) le système opérationel, c'est-à-dire la hiérarchie traditionnelle de l'autorité et des rôles; b) le système représentotif, c'est-ò-dire, un groupe de délégués élus par les trovailleurs pour représenter les points de vue des électeurs dans les comtés et les conseil: c) un système législatif, c'est-à-dire, "les conseils... dans lesquels les systèmes opérationnels et représentatifs se rencontrent et au moyen desquels chaque membre peut contribuer à formuler une politique et à évaluer les résultats découlont de l'application de cette politique. \$
Brown reconnoit aussi une procédure de recours que peut utiliser chaque membre de l'entreprise contre toute décision de son chef. Le grief est présenter à un niveou supérieur jusqu'au directeur général, si nécessaire. II existe aussi une cour d'oppel

Lo pensée de Brown s'insère dans un effort de "démocratiser " la direction et l'organisation de l'entreprise en rendant chocun conscient de son influence réelle et de la contribution possible qu'il peut opporter.

\section{Laurent BEL_ANGER}

\section{Group Thinking and Conference Leadership, William E. Utterback, New York: Holt,} Rinehart and Winston, Inc., 1964, 244 pp.

Cet ouvrage est un petit monuel pratique des techniques de discussion, il contient en effet une foule de conseils et de règles très simples découlant de l'observation $\in t$ du sens commun.

L'outeur dans la première partie: : Techniques of Discussion répond à la question suivante: Quand la discussion est-elle rentable? II faut selon Utterback un ensemble de conditions physiques favorables, un groupe bien constitué, un sujet de discussion qui soit odapté d̀ ce même groupe et une documentation appropriée. Viennent ensuite des chapitres où l'auteur indique comment se préparer, participer et servir de modérateur dans une discussion.

«Thinking straight , seconde portie de l'ouvroge, est composé de deux chapitres. Dans le premier l'auteur distingue trois types d'arguments basés sur l'oppel au principe, ou fait et à l'outorité. Viennent ensuite quelques principes: il fout éviter d'être trop émotif, timide, égoïste ou indolent et autres défauts qui risquent d'empêcher le groupe d'arriver ou consensus.

En troisième lieu l'auteur aborde différents genres de discussions: la table ronde communautaire, la discussion rodiodiffusée ou télévisée, lo conférence d'uffaires et l'assemblée délibérante. Ces chapitres contiennent en premier lieu une brève analyse du genre de débat, suivent une série de prescriptions et de conseils pratiques qui focilitent lo discussion.. 
En oppendice l'auteur présent des cas de discussions dont l'utilisaton est focilitée por l'e Instructor's Manual , qui acompagne cet ouvroge. On trouve oussi en appendice deux exemples concrets tirés des situations types. sont appliqués les principes exposés dans les chapitres précédents.

Cet ouvroge est de lecture et de compréhension fociles, en effet les principes et énoncés théoriques sont illustrés par des exemples concrets tirées des situations types. II s'agit donc d'un d'un ensemble de recettes et de règles qui ne peuvent être ignorées de ceux qui sont appelés d̀ travailler en comité. Ce monuel toutefois s'ovèrera surtout utile pour ceux qui n'ont aucune expérience des groupes de discussions, une lecture ropide de cet ouvroge suffirait d combler cette locune, car on trouve ici un ensemble de connaissonces utiles, facilement et rapidement accessibles.

\section{Jean MICHAUD}

Issues in Business and Society : Reading and Coses, by William T. Greenwood, Houghton Mifflin Company, Boston, 1964, 554 pages.

Cet excellent ouvroge n'apportera rien de nouveou à quelques lecteurs très au fait du sujet discuté. En effet, comme son titre l'annonce, il comprend une collection d'articles et de cas traitant chacun à son point de vue le thème très vaste de l'entreprise, des offaires et la société. Même pour les lecteurs privilégiés dont nous venons de parler, chaque article déjà lu isolément prendro peut-être un sens nouveau, plus riche et plus significatif, en tant que partie logique d'un ensemble cohérent.

Aux autres personnes intéressées, ces lectures offriront une matière substantielle, diversifiée et étendue propre d̀ les foire réfléchir sur les principoux points de vue des relotions entre l'entreprise et la société. Le thème général est onalysé en dix-neuf sujets différents dont, d'oprès $M$. Greenwood lui-même les deux-tiers sont controversés et l'autre tiers présente des tendances nouvelles, en oppostion ovec les protiques classiques traditionnelles des affaires.

Dans une première partie portant sur notre société de l'entreprise privée, nous pouvons lire des opinions évoluées et justes, d̀ notre ovis, concernant le copitolisme et lo liberté, l'entreprise, lo concurrence et le gouvernement, puis finalement le rôle des profits.
En deuxième et troisième lieu, $M$. Greenwood a sélectionné des articles traitant de l'entreprise et ses publics, et ensuite de l'entreprise et l'individu dans la société.

L'outre partie comprend des articles qui intéresseront tout particulièrement la plupart des lecteurs de cette Revue. II s'agit en effet des relations patronales-ouvrières; on $y$ porle de convention collective, de responsabités des dirigeants vis-à-vis l'emploi (pleinemploi et chômoge), et enfin des justes pratiques d'emploi.

Des orticles et des cas sur l'éthique, lo religion et l'entreprise forment la cinquième partie. Dans la suivante, on peut y lire d'excellents articles, entre autres, de Neil Chomberlain, Jomes K. Dent et Peter F. Drucker, où l'on essaie de dégoger les tendances vers une philosophie de la direction. Une dernière partie traite des responsobilités sociales dans l'entreprise.

Certes, les lecteurs de cet ouvrage ne seront pas tous d'accord avec $M$. Greenwood sur le choix des articles et des cas. Le caractères plus ou moins controversé des sujets traités, donne à la sélection une importance particulièrement significotive, car elle doit se faire parmi des tendances souvent diamétralement opposées. On peut finir en disant qu'une allure progressiste se dégage de cette excellente sélection d'articles et de cos, qui est certes susceptible d'intéresser un grand nombre de lecteurs.

\section{Bertrand BELZILE}

Emploi en France, par Henri Hatzfeld et Jocques Freyssinet, dans la Collection a Economie et Humanisme ", les Editions Ouvrières, Paris, 1964, 271 pages.

Considérant que l'emploi est ò l'origine de nombreux et grands conflits contemporains, les auteurs ont voulu présenter un livre de vulgarisation sur la situation octuelle dans leur pays, ainsi que sur certains problèmes connexes, comme la formation professionnelle, migration régionales, exode rurale et quelques autres. Ce présent ouvrage ne prétend pas aider les spécialistes de la question, mais il entend servir les travailleurs eux-mêmes et les étudionts intéressés à l'emplois en Fronce

L'ouvrage se divise en trois parties. Dans la première, sous le titre I'Equilibre Global de I'Emploi, les auteurs envisagent le chômage et le plein emploi au double aspect de la pensée économique et de l'expérience his- 